\title{
ORIGINAL ARTICLE Content validity of the Work Rehabilitation Questionnaire- Self-Report Version WORQ-SELF in a subgroup of spinal cord injury patients
}

\author{
R Portmann Bergamaschi ${ }^{1,2}, \mathrm{R}$ Escorpizo ${ }^{1,3,4}, \mathrm{~S}$ Staubli $^{5}$ and ME Finger ${ }^{1,3}$
}

Study design: Mixed methods study design.

Objectives: The Work Rehabilitation Questionnaire-Self-Report Version (WORQ-SELF) is an assessment tool based on the International Classification of Functioning, Disability and Health (ICF) developed to evaluate functioning in different patient populations in vocational rehabilitation (VR) settings. The objective of this study is to establish the content validity of WORQ-SELF in a subgroup of spinal cord injury (SCI) patients in the early post-acute context.

Setting: Swiss Paraplegic Research and Swiss Paraplegic Centre, Nottwil, Switzerland.

Methods: Contents of WORQ-SELF were compared with semi-guided interviews with SCI patients in Switzerland, the Comprehensive ICF Core Set for SCl early post-acute, and outcome instruments used in VR and $\mathrm{SCl}$. A frequency analysis was performed.

Results: WORQ-SELF represented 46 different ICF categories and of these 37 categories were confirmed by the patient interviews. The Comprehensive ICF Core Set for SCl confirmed 25 categories. Four instruments used in VR and SCl setting were identified. Contents of those instruments confirmed 14 categories of WORQ-SELF. Overall, 26 categories of the WORQ-SELF were confirmed by at least 2 of the 3 sources, 13 categories by 1 source and 7 were not confirmed by any of the sources.

Conclusion: The WORQ-SELF proved to have content validity for utility in patients with SCI within the context of VR. WORQ-SELF can be used to assess the functioning and disability of patients in the return to work process.

Spinal Cord (2014) 52, 225-230; doi:10.1038/sc.2013.129; published online 19 November 2013

Keywords: employment; work rehabilitation; vocational rehabilitation; ICF Core Sets

\section{INTRODUCTION}

Rehabilitation in spinal cord injury (SCI) is crucial to maximize the physical functioning, to prevent secondary complication and to facilitate reintegration in the community and in society. Most of the patients with traumatic SCI are young ${ }^{1}$ and perceive that they are able to work. ${ }^{2}$ However, involvement in employment is significantly lower than that observed in the population without disabilities. ${ }^{2}$ Hence, it becomes essential that work participation in SCI needs to be addressed and work disability be mitigated.

The quality of life in people with SCI depends, among other things, on vocational reintegration. ${ }^{3}$ The early expectations of a person with SCI are an important indicator of successful reintegration. ${ }^{4}$ Therefore, the period at the end of the first rehabilitation (early post-acute context) becomes critical in initiating steps to start vocational rehabilitation (VR). VR is a key process in work disability management to engage or re-engage individuals in work participation and employment. ${ }^{5}$ From the social context, VR is designed to maximize work participation of persons with disabilities and to promote their full integration and participation in the society. ${ }^{6}$ However, VR is a complex process as return to work is a complex outcome. To address this complexity, numerous health status measures are used to assess the functional limitations associated with SCI. However, most of these measures cover only limited aspects of the SCI-related experience of work and vary considerably with regard to content and psychometric properties. ${ }^{7}$ This degree of variation presents a number of challenges to professionals who would like to compare or transfer patient data across the continuum of care.

The International Classification of Functioning, Disability and Health (ICF) is a universal conceptual framework and classification system. ${ }^{8}$ Functioning is a result of the interplay between body structures and body functions at the body level and activities and participation at the community and social level, taking into account the personal and environmental factors. ICF can be used across different health settings; hence, its utility can facilitate comparison of outcome data across successive phases of the continuum of care and interventions in SCI. ${ }^{9}$ In 2010, the ICF Core Set for VR was developed. ${ }^{10}$ It has 90 ICF categories relevant in assessing functioning and disability in VR. As the ICF Core Set only provides 'what' to assess, it becomes imperative to develop an instrument of 'how' to assess. At the time, there had been no ICF-based questionnaire specifically developed to capture functioning in vocational settings. Hence, the Work Rehabilitation Questionnaire (WORQ) was developed based on the Brief ICF Core Set for VR. The

${ }^{1}$ Swiss Paraplegic Research (SPF), ICF Unit, Nottwil, Switzerland; ${ }^{2}$ School of Health Professions, Institute of Physiotherapy, Zurich University of Applied Sciences, Winterthur, Switzerland; ${ }^{3}$ ICF Research Branch, WHO FIC CC in Germany (at DIMDI), Nottwil, Switzerland; ${ }^{4}$ Department of Physical Therapy, Louisiana State University Health Sciences Center, New Orleans, LA, USA and ${ }^{5}$ Swiss Paraplegic Centre SPZ, Institute for Vocational Guidance, Nottwil, Switzerland 
initial version of WORQ was interview administered, its psychometric properties (test-retest reliability, internal consistency, construct/ content validity and feasibility) have been established in a population of patients with various health conditions in a return to work program. ${ }^{11}$ To promote better practicability and feasibility in the clinical setting, a self-reported version (WORQ-SELF) that contains 40 functioning questions and 18 socio-demographic and work-related questions representing a total of 46 ICF categories was developed. WORQ-SELF can be accessed at http://www.myworq.org/. As a psychometric property fundamental and essential to any measurement, content validity requires relevance of a construct to the intended setting and purpose of an instrument and may be evaluated by experts. ${ }^{12}$ To date, the content validity of WORQ-SELF in the context of people with SCI in the early post-acute phase has yet to be validated.

Hence, the objective of this study is to establish the content validity of the WORQ-SELF in the early post-acute context in SCI population in VR.

\section{MATERIALS AND METHODS}

Mixed methods were employed to study the content validity of the WORQSELF in the SCI population in the early post-acute phase by comparing the contents of WORQ-SELF with three different sources: individual patient interviews, the Comprehensive ICF Core Set for SCI and assessment instruments used in VR and SCI (Figure 1: Method synopsis). The ICF was used as the common taxonomy for content comparison across all three sources. The interviews and instruments were linked to the ICF, using published linking rules $^{13}$ while the ICF Core Set is already presented in terms of ICF categories. All comparisons were performed at the second level of the ICF categories.

\section{Phenomenological study using semi-guided interviews}

Semi-guided interviews with SCI patients using five ICF-based questions were conducted by one interviewer in an SCI clinic in Switzerland (will be called 'study center' from now on) from August 2012 to February 2013. Phenomenological analysis, a popular approach in psychology and nursing, looks at the subjective states and an insider's perspective, and is often combined with elements of content analysis. ${ }^{14}$

Study population. A subgroup of the general population, the study included patients with SCI in the post-acute context. This is the period at the end of first rehabilitation and when VR already began. Sampling in qualitative research is concerned with the richness of information and the number of participants required, therefore the full spectrum of individual factors should be represented and samples should consist of participants who best represent the research topic ${ }^{15}$ (Table 1).

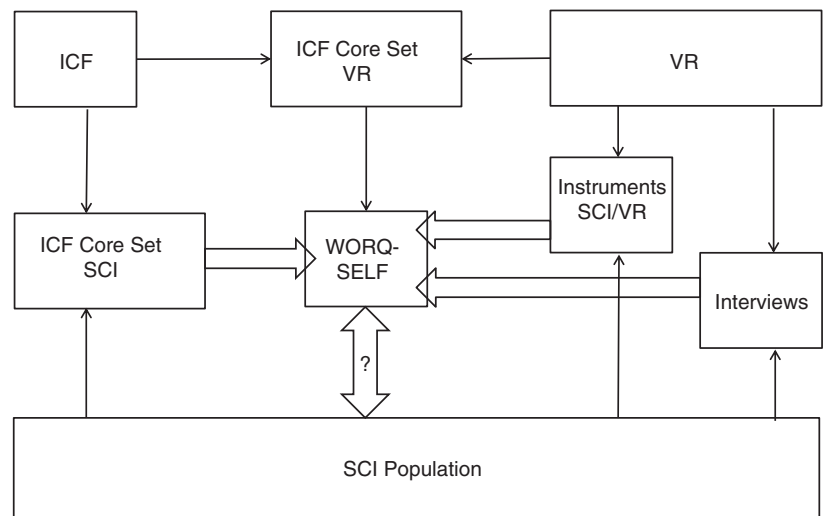

Figure 1 Method synopsis. ICF, International Classification of Function, Disability and Health; SCI, spinal cord injury; VR, vocational rehabilitation; WORQ-SELF, Work Rehabilitation Questionnaire-Self-Report Version.
Inclusion criteria for participants included a diagnosis of SCI, must be participating in VR, is age 18 years or older, proficient in German, has no intellectual disability, has been informed of the purpose of and reason for the study, and has signed the informed consent form. Sample size depended on whether saturation was reached. Saturation is the point at which additional interviews are not expected to yield new or valuable information and when depth and breadth of information is achieved. ${ }^{15}$ Saturation was expected to be reached with about 7 to 10 patient interviews. ${ }^{16}$

Data collection procedure of the qualitative study. A case report form was administrated to collect socio-demographic, health and work related data. To guide the personal interview, five open questions, similar to the questions used by Glässel et al $(2011)^{17}$ in the focus group study to explore the lived experiences of persons in VR with regard to functioning and contextual factors related to the ICF components were used. They addressed body function, activity and participation, environmental factors (barriers and facilitators) and personal factors (Table 2).

The study was approved by the Ethical Board of the Canton of Lucerne and conducted in compliance with the Declaration of Helsinki. The recruitment of patients was initiated by health professionals in the VR department of the study center in collaboration with a physician. After giving written informed consent one trained interviewer performed the interviews. The interviews were audio recorded.

Analysis of the qualitative study. The interviewer transcribed the interviews in verbatim and from which concepts were identified. These concepts were 'linked' to the best-fitting ICF categories. To check the quality of the linking process, $10 \%$ (randomly selected) of the concepts were linked independently by a second linker. Inter-linker agreement between the two linkers was established using kappa statistics. The obtained ICF categories were compared with the ICF categories of WORQ-SELF

\section{Table 1 Characteristics of participants $(N=9)$}

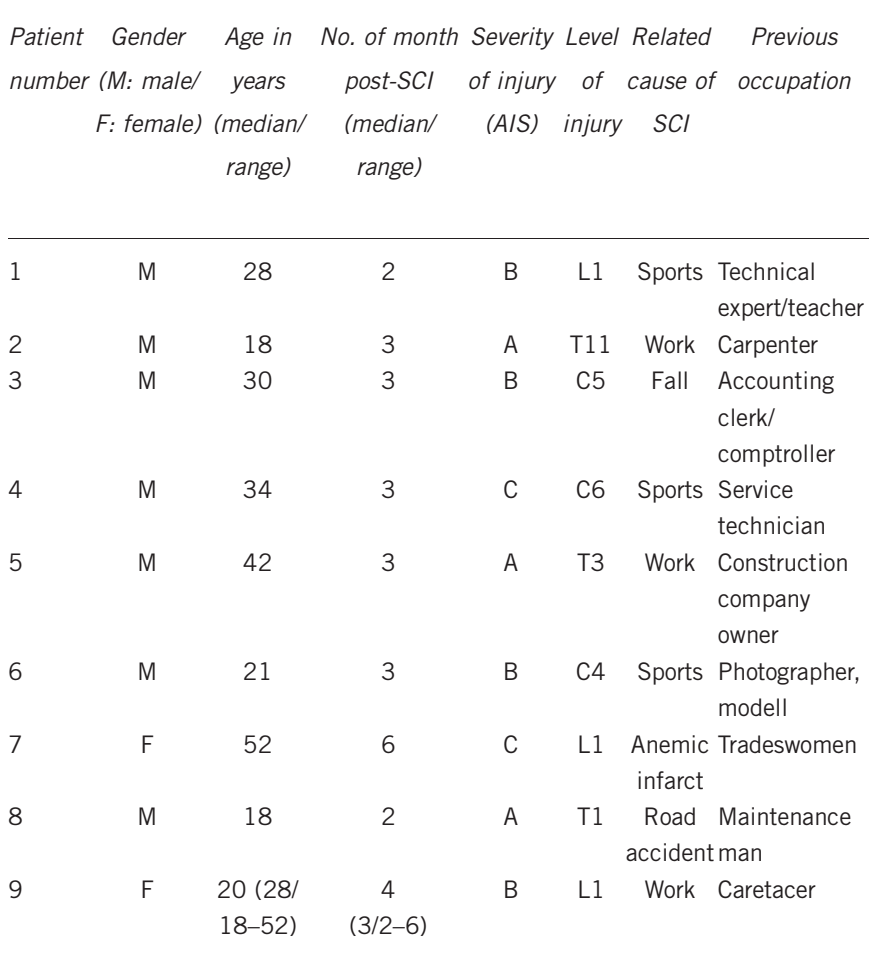

Abbreviations: AIS, ASIA (American Spinal Injury Association) Impairment Scale grade; A, complete: no motor or sensory function is preserved in the sacral segments S4-S5; $\mathrm{B}$, incomplete: sensory but not motor function is preserved below the neurological level and includes the sacral segments S4-S5; C, incomplete: motor function is preserved below the neurological level, and more than half of key muscles below the neurological level have a muscle grade less; Level of injury: L, lumbar; T, thoracic; C, cervical; SCI, spinal cord injury. 
Table 2 Open-ended questions of the patient interview

1. If you think about your body and mind, what does not work the way it is supposed to in relation to your participation in the vocational rehabilitation program? (body functions)

2. If you think about your daily life, what are you facing in relation to your participation in the vocational rehabilitation program? (activities and participation)

3. If you think about your environment and your living conditions, what barriers do you experience in relation to your participation in the vocational rehabilitation program? (environmental factors-barriers)

4. If you think about your environment and your living conditions, what do you find helpful or supportive in relation to your participation in the vocational rehabilitation program? (environmental factors-facilitators)

5. If you think about yourself, what is important about you and the way you handle your situation of vocational rehabilitation? (personal factors)

Note: standardized order of questions applied in an official German translation.

\section{Comparison of the Comprehensive ICF Core Set for SCI with the WORQ-SELF}

The ICF categories of the WORQ-SELF were compared with the Comprehensive ICF Core Set for SCI in the early post-acute context. It contains 162 second-, third- and forth-level categories. ${ }^{18}$ The extent to which WORQ-SELF captures the ICF categories from the ICF Core Set for SCI in the early postacute context was assessed.

\section{Comparison of assessment measures used in VR and SCI with the WORQ-SELF}

Contents of standardized instruments used in both VR and SCI rehabilitation were first identified compared with the WORQ-SELF (Table 3). These instruments were identified based on the systematic reviews conducted in the context of the development of the ICF Core Sets of $\mathrm{SCI}^{19}$ and $\mathrm{VR}^{5}$ and have already been previously linked to the ICF. Contents of the instruments and the WORQ-SELF were compared.

\section{Frequency analysis of the three sources}

A frequency analysis of the ICF categories from the interview, the ICF Core Set for SCI and the identified four standardized instruments with the WORQ-SELF was conducted to examine content validity. Content validity of each ICF category was established if the respective category from WORQ-SELF was identified in at least two of the three sources. This was an arbitrary cutoff decision we made.

\section{RESULTS}

\section{WORQ-SELF and interview contents}

Characteristics of the interviewed patients are presented in Table 1. In the saturation process patient's statements added up to 115 ICF categories. Saturation was reached after nine interviews (Figure 2: Saturation process). Thirty-seven of forty-six ICF categories $(80 \%)$ of WORQ-SELF were also indicated in at least one of the nine interviews. Nine categories of WORQ-SELF were not confirmed by the interviews: b160 Thought functions, b210 Seeing functions, b230 Hearing functions, d160 Focusing attention, d166 Reading, d210 Undertaking a single task, $\mathrm{d} 315$ Communicating with receiving nonverbal messages, d270 Complex interpersonal interactions, and d855 Non-remunerative employment (Table 4). The inter-linker agreement indicated an overall kappa of 0.82 (confidence interval: $0.75,0.89$ ).

WORQ-SELF and the Comprehensive ICF Core Set for SCI

Twenty-five of the forty-six ICF categories (54\%) of the WORQ-SELF were confirmed by the Comprehensive ICF Core Set for SCI (Table 4).

\section{WORQ-SELF and standard instruments}

Out of 106 instruments, four questionnaires were selected for the content comparison with WORQ-SELF, that were used in SCI rehabilitation as well as in VR setting. ${ }^{5,19}$ The four questionnaires include the Short Form $(\mathrm{SF} 36)^{20}$ to address the concept of healthrelated quality of life, McGill Pain Questionnaire ${ }^{21}$ to address pain and the Hospital Anxiety and Depression Scale ${ }^{22}$ and Beck Depression Inventory ${ }^{23}$ to address depression and anxiety (Table 3 ). Fourteen of the forty-six ICF categories (30\%) of the WORQ-SELF were confirmed by the four questionnaires (Table 4).

\section{Frequency analysis}

In all, 11 of the 46 ICF categories (24\%) in the WORQ-SELF were confirmed by all three sources and $15(33 \%)$ were named twice (for a total of 26 (57\%) WORQ-SELF categories named at least twice). Thirteen of the forty-six categories (28\%) were identified in only one source. Seven categories (15\%) were not contained in any of the studies: b210 Seeing functions, b230 Hearing functions, d160 Focusing attention, d166 Reading, d210 Undertaking a single task, d315 Communicating with receiving non-verbal messages and d720 Complex interpersonal interaction (Table 4).

The following five categories of the activity and the participation component were named in all three sources but were not included as a concept in WORQ-SELF: $\mathrm{d} 410$ Changing basic body position, $\mathrm{d} 445$ Hand and arm use, d460 Moving around in different locations, d640 Doing housework and d760 Family relationships.

The following four categories from environmental factors were named in the Comprehensive ICF Core Set for SCI and in at least eight interviews, but were not contained in WORQ-SELF: e115 Products and technology for personal use in daily living, e120 Products and technology for personal indoor and outdoor mobility and transportation, e150 Design, construction and building products and technology of buildings for public use, and e155 Design, construction and building products and technology of buildings for private use.

\section{DISCUSSION}

Our study examined the content validity of WORQ-SELF, an ICFbased self-reported generic questionnaire, to assess work-related functioning of patients with SCI in VR. Contents of WORQ-SELF were compared with three different sources: patient interviews, the ICF Core Set for SCI and standard questionnaires used in SCI and VR.

The interviews gave us a profound insight about the lived experience of the interviewed patients, therefore they weigh strongly. ${ }^{14}$ The same interviewer conducted all of the interviews to ensure consistency in the interview and evaluation techniques employed across the interviews. The inter-linker agreement with a kappa of 0.82 suggests that consistency was upheld. The ICF categories generated from the content of the interviews confirmed $80 \%$ of categories of WORQ-SELF. The results showed that the remaining $20 \%$ not mentioned by the interviewed patients reflected predominately sensory functions and cognitive skills. Contrary to the patient's statements, neuropsychological problems that have been described in the SCI population primarily related to executive functions like attention and concentration, ${ }^{24}$ in which the interviewed patients at the beginning of their VR process did not experience. Hence, it was not surprising that sensory functions and cognitive skills were not mentioned in the interviews. 


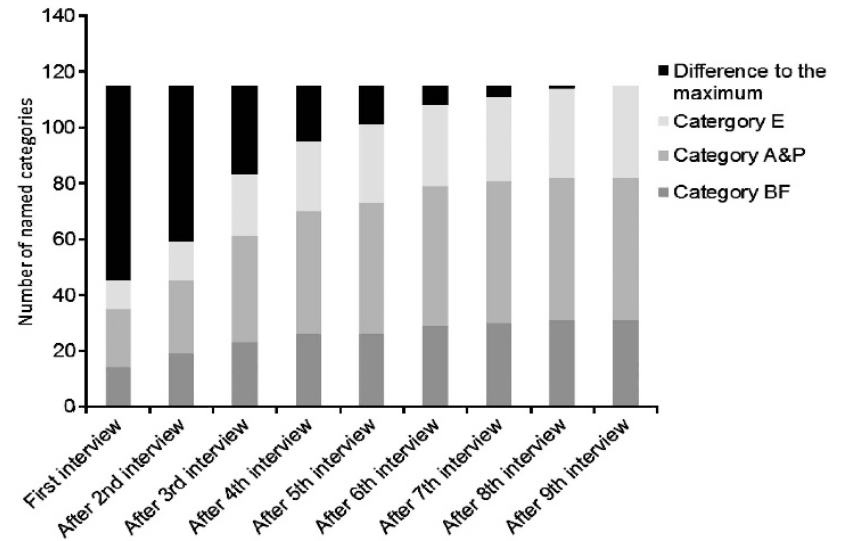

Figure 2 Saturation process. BF, body function; A\&P, activity and participation; $\mathrm{E}$, environmental factors.

The comparison of the Comprehensive ICF Core Set for SCI and WORQ-SELF showed a congruency of $54 \%$. While work-related concepts such as d825 Vocational training, d840 Apprenticeship, and d845 Acquiring, keeping and terminating a job were missing in the ICF Core Set for SCI, WORQ-SELF, in contrast, captures aspects of the VR process as intended, since it is based on the setting-specific ICF Core Set for VR.

With regard to the comparison between four instruments that are commonly used in both VR and rehabilitation of persons with SCI, the overall findings indicated a poor agreement of 14 of 46 categories (30\%). While 22\% ICF categories in SF36 were confirmed by WORQSELF, McGill Pain Questionnaire, Hospital Anxiety and Depression Scale and Beck Depression Inventory had categories mostly related to the ICF component of body function (Table 3). This finding is understandable considering that these four instruments are generic and not specifically developed for use in rehabilitation of SCI patients nor for VR. If these generic instruments are to be used within the context of VR and SCI, then it would be beneficial if a work-specific instrument like WORQ-SELF is used in parallel. WORQ-SELF ensures adequate coverage of activities and participation domains that are essential for designing appropriate work-related intervention. ${ }^{11}$

In the frequency analysis the categories of WORK-SELF that were not confirmed by any of the three sources belong mainly to mental and sensory functions, learning knowledge and communication and major life areas (education, and work and employment), corresponding to the missing categories in the patient interviews and the ICF Core Set for SCI. ${ }^{18}$ Even if the missing categories played a limited to no role in the lives of the interviewed patients, these areas of functioning may be relevant for the content validity of WORQSELF for the general population.

The five categories identified in all three sources that are not covered by WORQ-SELF represent aspects of activities and participation that SCI patients deal with on an everyday basis. These categories could be added to round out an SCI-specific version of WORQ-SELF.

Although the environmental factors category of e120 Products and technology for personal indoor and outdoor mobility and transportation and e150 Design, construction and building products and technology of buildings for public use were named in two sources and were considered as highly relevant in the interviews (eight of nine patients), they are not covered by WORQ-SELF. The findings suggest that these particular environmental factors would be important to consider to ensure successful work participation of persons with SCI. ${ }^{25}$ In addition to these environmental factors, the patient's
Table 3 Instruments used in $\mathrm{SCl}$ and in vocational rehabilitation

\begin{tabular}{|c|c|c|c|c|c|}
\hline $\begin{array}{l}\text { Name of } \\
\text { questionnaires }\end{array}$ & ICF concepts & $\begin{array}{l}S F- \\
36\end{array}$ & $M P Q$ & HADS & $B D I$ \\
\hline No. of items & & 36 & 11 & 14 & 21 \\
\hline \multicolumn{6}{|l|}{ ICF codes } \\
\hline b1260 & Extraversion & & & & $x$ \\
\hline b1263 & Psychic stability & & & & $x$ \\
\hline b1300 & Energy level & $x$ & & $x$ & $x$ \\
\hline b1302 & Appetite & & & & $x$ \\
\hline b134 & Sleep functions & & & & $x$ \\
\hline b152 & Emotional functions & $x$ & & & $x$ \\
\hline b1520 & Appropriateness of emotion & & & $x$ & \\
\hline b1522 & Range of emotion & & & $x$ & \\
\hline b1528 & Emotional functions, other spec. & & & $x$ & \\
\hline b1602 & Content of thought & & & $x$ & $x$ \\
\hline b1801 & Body image & & & $x$ & \\
\hline b280 & Sensation of pain & $x$ & $x$ & & \\
\hline b28010 & Pain in head and neck & & $x$ & & \\
\hline b28012 & Pain in stomach or abdomen & & $x$ & & \\
\hline b289 & Sensation of pain, other & & $x$ & & \\
\hline b530 & Weight maintenance functions & & $x$ & & \\
\hline b6400 & Functions of sex. arousal phase & & $x$ & & \\
\hline d177 & Making decisions & & & & $x$ \\
\hline d230 & Carrying out daily routine & $x$ & & & \\
\hline d4 & Mobility & $x$ & & & \\
\hline d4102 & Kneeling & $x$ & & & \\
\hline d4105 & Bending & $x$ & & & \\
\hline d430 & Lifting and carrying objects & $x$ & & & \\
\hline d4300 & Lifting & $x$ & & & \\
\hline d4451 & Pushing & $x$ & & & \\
\hline $\mathrm{d} 4500$ & Walking short distances & $x$ & & & \\
\hline d4501 & Walking long distances & $x$ & & & \\
\hline d4551 & Climbing & $x$ & & & \\
\hline d4552 & Running & $x$ & & & \\
\hline d5101 & Washing whole body & $x$ & & & \\
\hline d540 & Dressing & $x$ & & & \\
\hline d640 & Doing housework & $x$ & & & \\
\hline d750 & Informal social relationships & $x$ & & & \\
\hline d7500 & Informal rel.ships with friends & $x$ & & & \\
\hline d7501 & Informal rel.ships with neighbors & $x$ & & & \\
\hline d760 & Family relationships & $x$ & & & \\
\hline d850 & Remunerative employment & $x$ & & & $x$ \\
\hline d855 & Non-remunerative employment & & & & $x$ \\
\hline d9 & Community, social + civic life & $x$ & & & \\
\hline d9201 & Sports & $x$ & & & \\
\hline d9205 & Socializing & $x$ & & & \\
\hline d9208 & $\begin{array}{l}\text { Recreation + leisure, other } \\
\text { specified }\end{array}$ & & & & $x$ \\
\hline
\end{tabular}

Abbreviations: ICF, International Classification of Functioning, Disability and Health; BDI, Beck Depression Inventory; HADS, Hospital Anxiety and Depression Scale; MPQ, McGill Pain Questionnaire; SCI, spinal cord injury; SF-36, Short Form SF 36.

physical environment like accessibility (transportation and buildings) and work place adaptation can also been seen as important factors for consideration in the return to work process. ${ }^{25}$

\section{Limitations}

The impact of the interviewer on the content of the statements should be reflected upon. Even though the interview procedure was 
Table 4 Frequency analysis comparing WORQ-SELF contents versus three sources

\begin{tabular}{|c|c|c|c|c|c|c|}
\hline ICF codes & ICF concepts & WORQ-SELF & Interviews & ICF Core Set for SCI & Instruments & No. of agreement with WORQ-SELF \\
\hline b126 & Temperament and personality functions & $x$ & $x$ & $x$ & $x$ & $3^{*}$ \\
\hline b134 & Sleep functions & $x$ & $x$ & $x$ & $x$ & $3^{*}$ \\
\hline b144 & Memory functions & $\mathrm{x}$ & $\mathrm{x}$ & & & 1 \\
\hline b152 & Emotional functions & $x$ & $x$ & $x$ & $x$ & $3^{*}$ \\
\hline b210 & Seeing functions & $x$ & & & & 0 \\
\hline b230 & Hearing functions & $x$ & & & & 0 \\
\hline b235 & Vestibular functions & $\mathrm{x}$ & $\mathrm{x}$ & & & 1 \\
\hline b280 & Sensation of pain & $x$ & $x$ & $x$ & $x$ & $3^{*}$ \\
\hline b455 & Exercise tolerance functions & $x$ & $x$ & $x$ & & $2^{*}$ \\
\hline b730 & Muscle power functions & $x$ & $x$ & $x$ & & $2^{*}$ \\
\hline d177 & Making decisions & $x$ & $x$ & & $x$ & $2^{*}$ \\
\hline d210 & Undertaking a single task & $x$ & & & & 0 \\
\hline d230 & Carrying out daily routine & $x$ & $x$ & $x$ & $x$ & $3^{*}$ \\
\hline d240 & Handling stress and other psychological demands & $x$ & $x$ & $x$ & & $2^{*}$ \\
\hline d315 & Communicating: non-verbal messages & $x$ & & & & 0 \\
\hline d350 & Conversation & $x$ & $x$ & & & 1 \\
\hline d360 & Using communication devices and techniques & $x$ & $x$ & $x$ & & $2^{*}$ \\
\hline$d 430$ & Lifting and carrying objects & $x$ & $x$ & $x$ & $x$ & $3^{*}$ \\
\hline d440 & Fine hand use & $x$ & $x$ & $x$ & & $2^{*}$ \\
\hline$d 450$ & Walking & $x$ & $x$ & $x$ & $x$ & $3^{*}$ \\
\hline d455 & Moving around & $x$ & $x$ & $x$ & $x$ & $3^{*}$ \\
\hline $\mathrm{d} 470$ & Using transportation & $x$ & $x$ & $x$ & & $2^{*}$ \\
\hline d850 & Remunerative employment & $x$ & $x$ & $x$ & $x$ & $3^{*}$ \\
\hline d855 & Non-remunerative employment & $x$ & & & $x$ & 1 \\
\hline $\mathrm{d} 870$ & Economic self-sufficiency & $x$ & $x$ & $x$ & & $2^{*}$ \\
\hline e310 & Immediate family & $x$ & $x$ & $x$ & & $2^{*}$ \\
\hline e330 & People in positions of authority & $x$ & $x$ & $x$ & & $2^{*}$ \\
\hline e570 & Social security services, systems and policies & $x$ & $x$ & $x$ & & $2^{*}$ \\
\hline e580 & Health services, systems and policies & $x$ & $x$ & $x$ & & $2^{*}$ \\
\hline e590 & Labor and employment services/systems/policies & $x$ & $x$ & & & 1 \\
\hline Total & & 46 & 37 & 25 & 14 & $26^{*}$ \\
\hline
\end{tabular}

Abbreviations: ICF, International Classification of Functioning, Disability and Health; SCI, spinal cord injury; WORQ-SELF, Work Rehabilitation Questionnaire-Self-Report Version.

The cutoff level for validation is reached with $3^{*}$ and $2^{*}$.

standardized as much as possible and the interviewer was trained in conducting semi-structured interviews, the actual execution of the patient interviews could have impacted on the results.

Moreover, since personal factors (pf) are yet to be classified in the ICF, the pf that were identified by the three sources limits our ability to make comparison in terms of personal factors. Some concepts were coded with 'not defined' (nd), for example: in the seventh interview: 'I am not able to do certain things anymore'. 'Things' are too broad term to be linked precisely. Some concepts were coded with 'not covered' (nc). They were referred to as 'not covered' concepts in established linking rules, for example, autonomy.

In addition, some categories indicated in all three sources were coded at the third or the fourth level. To enable comparability, the third- and fourth-level categories were transformed to the second level, thus making the corresponding categories less specific. As a result, some information may have been lost during the transformation. For example, in the second interview the patient stated: 'I'm in pain above and below the operating area (back).' The corresponding 
fourth-level category b28013 Pain in back was transformed to the second-level category b280 Sensation of pain. As a result, the detail about the location of the pain disappears.

Our findings support the content validity of WORQ-SELF for the subgroup of SCI patients in the early post-acute context. Most of the important aspects of needs and resources concerning VR in this subgroup were considered by the WORQ-SELF. In the near future, further utility of WORQ-SELF in SCI should also consider concepts that were identified as lacking in WORQ-SELF, for example, e120 Products and technology for personal indoor and outdoor mobility and transportation and e150 Design, construction and building products and technology of buildings for public use. Further studies to evaluate content validity in post-acute or chronic SCI patients engaged in VR are needed to gain evidence of the validity of the WORQ-SELF throughout the whole VR process across the health-care continuum.

\section{DATA ARCHIVING}

There were no data to deposit.

\section{CONFLICT OF INTEREST}

The authors declare no conflict of interest.

\section{ACKNOWLEDGEMENTS}

This study was funded by Swiss Paraplegic Research, Nottwil, Switzerland. This publication is the sole responsibility of the ICF Research Branch.

1 Wyndaele M, Wyndaele JJ. Incidence, prevalence and epidemiology of spinal cord injury: what learns a worldwide literature survey? Spinal Cord 2006: 44: 523-529.

2 Thomasson P, Post M, van Asbeck F. Return to work after spinal cord injury. Spinal Cord 2000; 38: 51-55.

3 Manns PJ, Chad KE. Components of quality of life for persons with a quadriplegic and paraplegic spinal cord injury. Qual Health Res 2001; 11: 795-811.

4 Schönherr MC, Groothoff JW, Mulder GA, Schoppen T, Eisma WH. Vocational reintegration following spinal cord injury: expectations, participation and interventions. Spinal Cord 2004; 42: 177-184.

5 Escorpizo R, Finger ME, Glassel A, Gradinger F, Luckenkemper M, Cieza A. A systematic review of functioning in vocational rehabilitation using the internationa classification of functioning, disability and health. J Occup Rehabil 2011; 21: 134-146.

6 Parker RM, Szymanski EM and Patterson JB (eds). Rehabilitation Counseling: Basics and Beyond, 4th edn. Pro-Ed: Austin, 2005
7 Dijkers M. Quality of life of individuals with spinal cord injury: a review of conceptualization, measurements and research findings. J Rehabil Res Dev 2005; 42: 87-110.

8 World Health Organization. International Classification of Functioning. Disability and Health. World Health Organization: Geneva, Switzerland, 2001.

9 Kirchberger I, Biering-Sorensen F, Charlifue S, Baumberger M, Campbell R, Kovindha A et al. Identification of the most common problems in functioning of individuals with spinal cord injury using the International Classification of Functioning, Disability and Health. Spinal Cord 2010; 48: 221-229.

10 Finger ME, Escorpizo R, Glassel A, Gmunder HP, Luckenkemper M, Chan C et al. ICF Core Set for vocational rehabilitation: results of an international consensus conference. Disabil Rehabil 2012; 34: 429-438.

11 Escorpizo R, Finger ME, Reneman MF. Integration and application of the International Classification of Functioning, Disability and Health (ICF) in return-to-work. In: Schultz IZ and Gatchel R (eds). Handbook of Return to Work: Springer: New York Heidelberg Dordrecht London, 2013.

12 Streiner D, Geoffry R. Health Measurements Scales, A Practical Guide to Their Development and Use, 4th edn. Oxford University Press: Oxford New York, 2008.

13 Cieza A, Geyh S, Chatterji S, Kostanjsek N, Ustun B, Stucki G. ICF linking rules: an update based on lessons learned. J Rehabil Med 2005; 37: 212-218.

14 Hancock B, Ockleford E, Windridge K. An introduction to qualitative research. East Midlands/Yorkshire \& Humber: NIHR Research design service for East Midlands, NIHR research design service for Yorkshire \& Humber 2009.

15 O'Reilly M, Parker N. 'Unisatisfactory Saturation': a critical exploration of the notion of saturated sample sizes in qualitative research. Qual Res 2013; 13: 190-197

16 Brod M, Tesler LE, Christensen TL. Qualitative research and content validity: developing best practices based on science and experience. Qual Life Res 2009; 18: $1263-1278$.

17 Glässel A, Finger ME, Cieza A, Treitler C, Coenen M, Escorpizo R. Vocational rehabilitation from the client's perspective using the International Classification of Functioning, Disability and Health (ICF) as a Reference. Occup Rehabil 2011 167-178.

18 Kirchberger I, Cieza A, Biering-Sørensen F, Baumberger M, Charlifue S, Post MW et al. ICF Core Sets for individuals with spinal cord injury in the early post-acute context. Spinal Cord 2010; 48: 297-304.

19 Post MW, Kirchberger I, Scheuringer M, Wollaars MM, Geyh S. Outcome parameters in spinal cord injury research: a systematic review using the International Classification of Functioning, Disability and Health (ICF) as a reference. Spinal Cord 2010; 48: 522-528.

20 Ware JE. SF-36 Health Survey. Manual and interpretation guide. New England Medical Center, Boston, MA: The Health Institute, 1997.

21 Melzack R. The McGill Pain Questionnaire: major properties and scoring methods. Pain 1975; 1: 277-299.

22 Zigmond AS, Snaith RP. The hospital anxiety and depression scale. Acta Psychiat Scand 1983; 67: 361-370.

23 Beck AT, Steer RA, Carbin MG. Psychometric properties of the Beck Depression Inventory: Twenty-five years of evaluation. Clin Psychol Rev 1988; 8: 77-100.

24 Murrey RF, Asghari A, Egorov DD, Rutkowski SB, Sidall PJ, Soden RJ et al. Impact of spinal cord injury on self-perceived pre- and postmorbid cognitive, emotional and physical functioning. Spinal Cord 2007; 45: 429-436.

25 Escorpizo R, Miller W, Trenaman L. Work and employment. In: Eng J, Teasell R, Miller W, Wolfe D, Townson A and Hsieh J et al. (eds). Spinal Cord Injury Rehabilitation Evidence. Vol 4.0. Vancouver, 2012, pp 1-22. 